\title{
Broschek, Jörg, und Patricia Goff (Hrsg.) (2020): The Multilevel Politics of Trade
}

\section{Toronto/Buffalo/London: University of Toronto Press. 400 Seiten. $\$ 49.95$}

\section{Henrik Scheller}

Angenommen: 23. September 2021 / Online publiziert: 13. Oktober 2021

(C) Der/die Autor(en) 2021

Dass neue Impulse für die Föderalismusforschung hierzulande nicht selten aus dem US-amerikanischen Raum kommen, beweist einmal mehr der neue, von Jörg Broschek und Patricia Goff herausgegebene Sammelband „The Multilevel Politics of Trade“. Denn das Verhältnis zwischen Föderalismus und Wirtschaft - einschließlich der neoliberalen Auswüchse der letzten 30 Jahre - sind in Deutschland schlichtweg ein Nichtthema. Dies ist umso problematischer, als ein nicht unwesentlicher Teil der föderalismustheoretischen Ansätze, mit denen in der Bundesrepublik bevorzugt operiert wird, nach wie vor durch ökonomisch inspirierte Ansätze und Annahmen mit einem Effizienz- und Wettbewerbsbias geprägt werden, die ihre Ursprünge ebenfalls in der amerikanischen Forschungstradition zu Mehrebenensystemen und der Problematik der öffentlichen Güter der 1950-/1960er-Jahre haben. Umso erfreulicher ist es, dass die Herausgeberin und der Herausgeber mit ihrer fokussierten Analyse von Beteiligungsformen und -verfahren subnationaler Einheiten an der Aushandlung von internationalen Freihandelsabkommen eine - wenn auch eng gezogene Schneise für sehr grundlegende wirtschaftspolitische Interaktionsfragen im globalen Mehrebenensystem einerseits und innerhalb einzelner föderaler Mehrebenensysteme andererseits schlagen und dafür rund zwei Dutzend ausgewiesene Autorinnen und Autoren gewinnen konnten. Auch jenseits der Föderalismusdimension ist dieser Ansatz verdienstvoll, bringt er doch ein wenig mehr Licht in die Blackbox der Verhandlungen internationaler Freihandelsabkommen, wie etwa CETA, TTIP, NAFTA 2.0 u. a., deren Zahl inzwischen weltweit bei über 260 liegen dürfte.

Broschek und Goff entwickeln in ihrer Einleitung einen ebenso einfachen wie überzeugenden analytischen Rahmen, der von drei zentralen Fragen angeleitet wird, um auf diese Weise drei Untersuchungsdimensionen berücksichtigen zu können. Den

Henrik Scheller $(\bowtie)$

Deutsches Institut für Urbanistik, Berlin, Deutschland

E-Mail: scheller@difu.de 
Ausgangspunkt dafür bildet die Frage nach den Mustern und Formen der Mitwirkung subnationaler Gebietskörperschaften in der internationalen Handelspolitik sowie etwaigen Schlüsselfaktoren, die die beobachtbaren Variationen im Engagement dieser Einheiten in verschiedenen Bundesstaaten einschließlich deren Auswirkungen erklären. Diese Grundüberlegungen spitzen Broschek und Goff auf die Frage zu, inwieweit wir derzeit möglicherweise einen größeren Transformationsprozess hin zu einer neuen, ebenenübergreifenden Handelsordnung beobachten können, in der subnationale Einheiten und Regionen zu zentralen Stakeholdern werden könnten. Dazu haben Herausgeberin und Herausgeber ihre Autorinnen und Autoren auf eine je länderspezifische Untersuchung verschiedener Perspektiven verpflichtet: ,the policy dimension, the institutional dimension, and the state-society dimension" (S. 6). Mit dieser Differenzierung wird nicht nur dem Umstand Rechnung getragen, dass sich das reine Ausmaß an Freihandelsabkommen und die damit verbundenen PolicyInhalte in den letzten Jahren deutlich verändert haben. Auch die Rolle der institutionellen Akteure, die in die Aushandlungsprozesse solcher Regime involviert sind, hat sich geweitet. In der Folge hat sich auch die gesellschaftliche Wahrnehmung entsprechender Policies und Regime gewandelt: Soziale Akteure sehen zunehmend ihre Verbraucherrechte berührt und verlangen entsprechende Anhörungs-, Mitsprache- und Vertretungsrechte - mit Auswirkungen auf die politischen Verhandlungsakteure. Substaatliche Einheiten in Föderalstaaten werden dabei von Broschek und Goff vor allem als Wahrer ihrer gesetzlich normierten Interessen gefasst, die auf eine Sicherstellung ihrer institutionellen Integrität bedacht sind. In diesem Sinne werden Verhandlungen von Freihandelsabkommen als Teil der internationalen Handelspolitik hier als eine föderale Kompetenzfrage zur Wahrung ökonomischer Interessen modelliert, die durch soziale Akteure und deren öffentlich-mediale Politisierung dieser Regime erst richtig getriggert wird.

Die Einzelbeiträge des Bandes widmen sich nicht nur ausgewählten Föderalstaaten, sondern beleuchten auch die Europäische Union als ,a distinct Federation“ (S. 281). Abschnitt 1 widmet sich aber zunächst den Verhandlungen der Freihandelsabkommen CUSFTA, CETA, TPP und TTIP in Kanada, den USA und Mexiko. In der Gegenüberstellung der Einzelanalysen wird deutlich, dass die kanadischen Provinzen - und allen voran natürlich Quebec - in den Verhandlungen der verschiedenen Handelsabkommen schon frühzeitig eine Chance zur Stärkung sowohl der eigenen Rolle und Kompetenzen als auch der intergouvernementalen Abstimmungs- und Konsultationsmechanismen erkannten und diese entsprechend nutzten. Demgegenüber zeichnen sich die USA seit jeher durch einen deutlich fragmentierteren Binnenmarkt als die EU aus, wie Michelle Egan in ihrem Beitrag konstatiert mit Folgen für einen deutlich selbstbewussteren Mitwirkungsanspruch der US-Bundesstaaten in entsprechenden Verhandlungen. Die Einzelanalysen im zweiten Abschnitt beleuchten die Föderalstaaten Schweiz, Deutschland, Österreich und Belgien sowie Australien. Die drei erstgenannten Föderalstaaten unterscheiden sich dabei allein schon aufgrund ihrer territorialen Größe, Wirtschaftskraft und bundesstaatlichen Genese, die in den letzten Jahrzehnten vor allem durch ihre Einbettung in einen forcierten Prozess der marktschaffenden Europäisierung geprägt war, deutlich von den Bundesstaaten des amerikanischen Kontinents. Wie auch in den anderen Länderstudien verdeutlichen Broschek, Peter Bußjäger und Christoph Schramek in 
ihrem vergleichenden Beitrag zu Österreich und der Bundesrepublik beispielhaft, dass die internationale Handelspolitik als "neues“ Politikfeld nicht allein - wie oft in der Literatur anzutreffen - durch den Bund bzw. den Zentralstaat als Möglichkeit zu einer Kompetenzausweitung erschlossen wird. Vielmehr nutzen auch die deutschen und österreichischen Länder neu entstehende Regelungsbedarfe in handelspolitischen Grauzonen, um eigene Zuständigkeiten abzustecken. Bevorzugtes Instrument hierfür sind vor allem die seit jeher ausgeprägten Mechanismen der horizontalen Selbstkoordination in Form verschiedener Ministerkonferenzen, die allerdings gleichzeitig dafür sorgen, dass die Rolle der Länder gegenüber dem Bund eher schwach bleibt. Ein dritter Abschnitt des Bandes widmet sich in vertiefenden Einzelanalysen der „state-society dimension“, indem die Handelspolitik der EU seit dem Lissabon-Vertrag, ebenenübergreifende Aspekte der Parteipolitik in der EU und der Bundesrepublik sowie die Rolle der Kommunen in entsprechenden Freihandelsverhandlungen beleuchtet werden.

Insgesamt liefert der Band von Broschek und Goff einen wichtigen Beitrag zum Verständnis - wenn auch eines sehr spezifischen, aber immer bedeutsamer werdenden Ausschnitts - der wirtschaftspolitischen Koordinierung innerhalb von Mehrebenensystemen. Weitere Forschungsarbeiten können und sollten hier anknüpfen. Das akkurat hergeleitete Untersuchungsdesign, das durch die pointierte Verdichtung der Ergebnisse in der Zusammenfassung von Broschek und Goff erste generalisierende Ansätze aufzeigt, kann hier durchaus von Nutzen sein. Wünschenswert wäre es beispielsweise, wenn in Zukunft noch stärker der Fokus auf die inhärent unitarisierenden Tendenzen von Wirtschaftspolitiken gerichtet wird. Inwieweit wird also mit Verweis auf eine vermeintlich notwendige Marktschaffung und Handelsliberalisierung - von welchen Akteuren eigentlich? - ein „Abbau“ oder zumindest eine Überwindung territorial begründeter Zuständigkeiten für föderale Teilmärkte gefordert? Zudem sollte der analytische Blick auf die „state-society dimension“ insofern geweitet werden, als auch der Einfluss von Unternehmen, Wirtschaftsverbänden und der ökonomischen Politikberatung in solchen Verhandlungen noch stärker Berücksichtigung findet. Denn gerade der deutsche Fall zeigt, dass diese Akteure mit einem Verweis auf die vermeintliche „Kleinstaaterei“ und zu viele länderspezifische Regelungen immer wieder ihre Forderungen nach bundeseinheitlichen Regeln begründen. Der Erhalt von Wirtschaftswachstum und Arbeitsplätzen erweist sich dabei noch immer als funktionierende Drohgebärde gegenüber Bund, Ländern und Kommunen, die sich darob nicht selten selber - ob bewusst oder nicht - als Unitarisierungstreiber gerieren. Auch bliebe zu prüfen, inwieweit sich die zunehmende Politisierung und Polarisierung von internationalen Freihandelsabkommen nicht nur als Auslöser einer föderalen Selbstbehauptung der subnationalen Einheiten modellieren lässt, sondern ggf. auch als Ausdruck einer Transformation hin zu konkurrierenden Wirtschaftsvorstellungen, die sich gerade bottum-up von den Kommunen und Regionen ausgehend in Gestalt neuer Formen der Kreislaufwirtschaft und der Gemeinwohlökonomie etablieren. Wie auch immer: Der Band von Broschek und Goff liefert in jedem Fall wertvolle Anstöße, um das Verhältnis von Föderalismus und Wirtschaftspolitik endlich intensiver zu diskutieren. 
Funding Open Access funding enabled and organized by Projekt DEAL.

Open Access Dieser Artikel wird unter der Creative Commons Namensnennung 4.0 International Lizenz veröffentlicht, welche die Nutzung, Vervielfältigung, Bearbeitung, Verbreitung und Wiedergabe in jeglichem Medium und Format erlaubt, sofern Sie den/die ursprünglichen Autor(en) und die Quelle ordnungsgemäß nennen, einen Link zur Creative Commons Lizenz beifügen und angeben, ob Änderungen vorgenommen wurden.

Die in diesem Artikel enthaltenen Bilder und sonstiges Drittmaterial unterliegen ebenfalls der genannten Creative Commons Lizenz, sofern sich aus der Abbildungslegende nichts anderes ergibt. Sofern das betreffende Material nicht unter der genannten Creative Commons Lizenz steht und die betreffende Handlung nicht nach gesetzlichen Vorschriften erlaubt ist, ist für die oben aufgeführten Weiterverwendungen des Materials die Einwilligung des jeweiligen Rechteinhabers einzuholen.

Weitere Details zur Lizenz entnehmen Sie bitte der Lizenzinformation auf http://creativecommons.org/ licenses/by/4.0/deed.de. 